\title{
Physical and Electrochemical Characterization of Palm Kernel Shell Biochar (PKSB) as Supercapacitor
}

\author{
Wan Azlina Wan Ab Karim Ghani ${ }^{1}$, Praveen Shawn Fernandez ${ }^{1}$, Mohamad Qayyum Halele ${ }^{1}$, Shafreeza Sobri ${ }^{1}$, Jasronita \\ jasni $^{2}$ \\ ${ }^{1}$ Department of Chemical and environmental Engineering , Faculty of Engineering, Universiti Putra Malaysia, Serdang, Selangor, \\ MALAYSIA \\ ${ }^{2}$ Department of Electrical and Electronic Engineering, Faculty of Engineering, Universiti Putra Malaysia, Serdang, Selangor, MALAYSIA
}

\begin{abstract}
A potential low cost and environmentally friendly supercapacitor has been prepared from Palm Kernel Shell Biochar (PKSB). In this study, physical and electrochemical properties of raw, activated and chemical treated (potassium hydroxide $(\mathrm{KOH})$ ) as supercapacitors such as high carbon content, high charge storage capacity and stable were evaluated. For physical analyses, the scanning electron microscopy (SEM) was used to study the surface morphology and surface area and porosity were measured using Brunaurer-Emmert-Teller (BET). The chemical treated PKSB shows the highest surface area values of $55.15 \mathrm{~m}^{2} / \mathrm{g}$ as compared to raw and activated samples with surface area are $0.17 \mathrm{~m}^{2} / \mathrm{g}$ and $19.32 \mathrm{~m}^{2} / \mathrm{g}$, respectively. This is verified by in enhancement of capacitance achieved from $1.76 \times 10^{-3} \mathrm{Fg}^{-1}$ for the activated biochar and $1.87 \times 10^{-6} \mathrm{Fg}^{-1}$ for untreated PKSB showed by Raman spectroscopy. This enhancement reflected the charge storage capacity is attributed to the creation of broad distribution in pore size and a larger surface area. In addition, this phenomenon also supported by the electrochemical profiles through cyclic voltammogram (CV) measured by Potentiostat-Gavanostat (EIS). CV of the treated PKSB gave better square shape than the activated and raw biochar samples. These characterizations conclude that the raw palm kernel biochar need further treatment to become supercapacitor electrodes to replace activated carbon.
\end{abstract}

\section{Introduction}

In response to the changing global landscape, energy has become a primary focus of the major world powers and scientific community. There has been great interest in developing and refining more efficient energy storage devices. One such device, the supercapacitor, has matured significantly over the last decade and emerged with the potential to facilitate major advances in energy storage.

Supercapacitors, also known as ultracapacitors are electrical energy storage devices. Compare to other electrical energy storage devices such as conventional capacitors, batteries, and fuel cells, supercapacitors can store high capacity electrical energy at respectively high power density $[1,2,3]$ as illustrated in Figure 1 . The charge-storage process for supercapacitor is highly reversible, resulting in longer cycle-life compare to other power storage devices [3]. Advanced carbon materials, such as grapheme, carbon nanotubes and carbon onions from metal carbide have shown high capacitance and high power density in electrochemical supercapacitors and hybrid supercapacitors. However, those advanced carbon materials will be significantly limited by high cost [4]. In addition, most conventional synthesis methods for these innovative carbon materials involve application of toxic strong oxidants, such as chlorine, potassium permanganate and hydrazine [5]. Therefore, hierarchical carbon materials, prepared from sustainable precursors with scalable processes, would be of great benefit to large scale application of supercapacitor in energy storage and conservation.

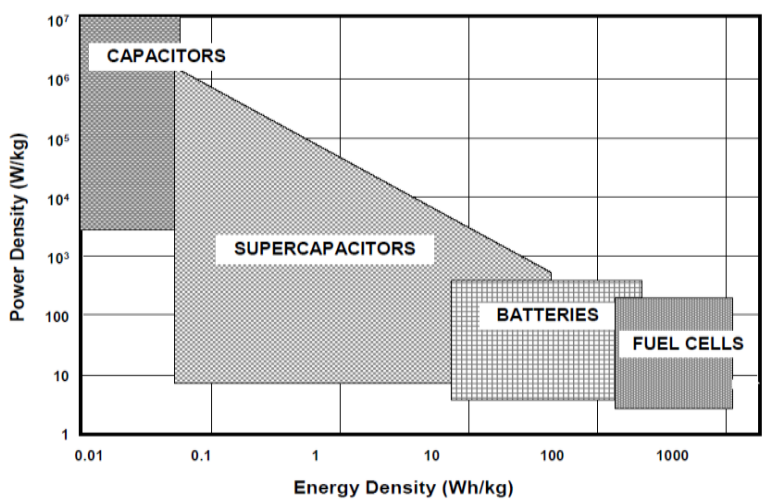

Figure 1. Ragone plot of energy storage devices [6]

The emerging studies shows an interest in agro-based carbon materials to replace the conventional activated carbon or even more, the advanced carbon material like graphene and carbon nanotube. This is because, agrobased materials are much cheaper, sustainable, and readily scalable in the mass market with safer 
synthesizing procedures options [7]. Some agro-based carbon materials (also called Biochar) have been proven to be promising options in producing relatively high specific surface area upon proper activation due to readily available pores in the microstructures. Biochar is a carbon-rich material produced through pyrolysis of biomass, such as wood, manure, and crop residue. Biochar has demonstrated promising potential in the field of supercapacitors. Some of the notable biomass precursors that have been prepared were corn grains [8], cypress [9], distillers dried grains with solubles (DDGS)[10], palm oil empty fruit bunch (EFB) [11], red cedar wood [12], rice husk, silk cocoon [13], and sunflower seed shell [14]. Ultimately, to achieve highly stable reversible electrical energy storage capacity and high power density in supercapacitors, carbon materials to fabricate electrodes, should possess a hierarchical mesoporous structure with high surface, high conductivity, suitable pore size distribution and long-term cyclability.

This paper aimed at comparing physical electrochemical characterization of palm kernel shellbiochar as supercapacitor electrodes. Malaysia being the second largest oil palm producer in the world with about 5 million hectare of oil palm plantation generates huge amount of oil palm biomass. Oil palm shell shell (PKS) is being generated as by-products from the 426 oil palm mills in Malaysia [15]. Scanning Electron Microscope (SEM),Brunaurer-Emmett-Teller (BET) surface area, Raman spectroscopy, Fourier Transform Infrared (FTIR), and potentiostat-galvanostat (EIS) have been be performed and evaluated to study their performance feasibility as compared to the conventional activated carbon.

\section{Materials and Methodologies}

For this study, three forms of Biochar samples for each organic materials are prepared, namely; control BC (raw) (not activated and not treated), activated BC (activated but not treated), and treated and activated BC (activated and treated). Biochar was prepared through pyrolysis of a PKS in a Nitrogen gas atmosphere by applying a temperature program to a MTI GSL-1100X tube furnace. The temperature was increased from room temperature to $200^{\circ} \mathrm{C}$ and then held at this temperature for $1 \mathrm{~h}$ to remove moisture. This was followed by increasing temperature to $750{ }^{\circ} \mathrm{C}$ at around $3{ }^{\circ} \mathrm{C} / \mathrm{min}$ and dwelling at this temperature for $1 \mathrm{~h}$ to remove volatile organics and to carbonize solid residues. The resulting Biochar chunk was removed from the furnace for characterization and evaluation after the temperature was cooled down to room temperature. The $\mathrm{BC}$ chunk was cut into small portions. Those with weights of approximately $1 \mathrm{mg}$ were directly used as supercapacitor electrodes without introducing any organic binder.

\subsection{Activation of biochar electrodes}

Biochar electrodes were further activated by immersing them into Potassium Hydroxide $(\mathrm{KOH})$ solution at room temperature overnight to increase surface hydrophilicity and the coverage of surface oxy-gen groups. They were rinsed using deionized before use. The activation process was carried out for three hours at $950{ }^{\circ} \mathrm{C}$ in a muffle furnace. The rate at which the muffle furnace heating is set is $5^{\circ} \mathrm{C} / \mathrm{min}$. Once the activation was completed, the activated $\mathrm{BC}$ was cooled within the muffle furnace in the same Nitrogen gas environment until the temperature drops to room temperature, which takes approximately nine hours. Then, the activated biochar sample is rinsed with $0.1 \mathrm{~mol} / \mathrm{L}$ hydrochloric acid $(\mathrm{HCl})$ at $100{ }^{\circ} \mathrm{C}$ using a spiral condenser. Once that is done, the activated $\mathrm{BC}$ is again rinsed this time with deionized water to $\mathrm{ph} 7$ (neutral). The reason for using deionized water specifically is to avoid contact of external ions on the surface of the activated $\mathrm{BC}$ which might sabotage the experimental results. Lastly, the activated BC sample is dried at $105{ }^{\circ} \mathrm{C}$ for the overnight under vacuum.

\subsection{Treatment of BC electrodes}

The activated BC samples were then further modified with $4 \mathrm{M} \mathrm{HNO}^{3}$. This is to facilitate for the third control sample, which is the activated and treated $\mathrm{BC}$ sample. The activated BC samples were left to soak in $20 \mathrm{~mL}$ of 4 $\mathrm{mol} / \mathrm{dm}^{3} \mathrm{HNO}_{3}$ solution sealed in Polytetrafluoroethylene (PTFE) reactor for 48 hours at $150{ }^{\circ} \mathrm{C}$. Once soaking was done, the treated $\mathrm{BC}$ was then cooled and filtered. The $\mathrm{BC}$ electrodes were rinsed with deionized water until the filter liquid equaled $\mathrm{pH} 7$. Lastly, the filter cake of treated $\mathrm{BC}$ was collected and left to dry overnight in a conventional oven at temperature $110{ }^{\circ} \mathrm{C}$.

\subsection{Physical characterization}

Surface morphology and elemental composition of the electrodes were characterized using a field-emission environmental scanning electron microscope operating at $15 \mathrm{keV}$ equipped with a light-element energy-dispersive spectroscope. BET surface areas of BC samples were measured using a Machine (Micromeritics Gemini VII 2390 V1.03) surface area/pore volume analyzer. Raman measurements were performed using a high-resolution research-grade (Horiba LabRAM HR 3D-capable)Raman spectroscopy imaging system that is optimized for the visible-to-NIR spectral range. A $532 \mathrm{~nm}$ wavelength laser was used in this study. DC electrical conductivity of BC was determined from Ohmic resistance, cross-section area, length and porosity of a small Palm Kernel Shell chunk based on Pouillet's law. The Ohmic resistance corresponds to the slope of linear potential-current curve measured by applying potential scan to two ends of the $\mathrm{BC}$ electrode chunk

\subsection{Electrochemical measurements}

Voltammetric and constant current charge-discharge studies of a single BC electrode were carried out in 0.5 mol solution with a coil of $1 \mathrm{~mm}$ diameter Platinum wire and a silver/silver chloride $(\mathrm{Ag} / \mathrm{AgCl})$ as the counter electrode and the reference electrode, respectively. The 
electrical contact of a BC electrode was obtained by wrapping fine gold wire around the PKS portion to minimize contact resistance. The geometric surface area is around 0.08 with $2 \mathrm{~mm}$ in width and $4 \mathrm{~mm}$ in length. The capacitive behavior of a BC supercapacitor constructed by putting two BC electrodes in similar size separated by a sheet of glassy filter-paper in 0.5 mol was evaluated by cyclic voltammetry and chronopotentiometry. Their preliminary durability was evaluated via successive potential cycling.

\section{Results and Discussion}

\subsection{Physical characterization of Palm Kernell Shell biochar.}

Figure 2 shows the result SEM of original PKS biochar, activated PKS biochar and treated PKS biochar, respectively. In these images, light region correspond to carbon wall and black region correspond to pores. The PKS biochar developed high porosity, presenting longitudinal pores with sizes ranging from micro to macro pores $(10-200 \mu \mathrm{m})$ [15]. It is clearly shows that the morphology of treated PKS biochar was more porous compared to original PKS biochar. The porous structures of the treated PKS Biochar sample indicate clearly that the potassium hydroxide, $\mathrm{KOH}$ activation process did in fact slice through the pores causing a larger pore size structure to be formed, and also the pore distribution is seen to be more frequent in an orderly manner. The positive results obtained from the difference in surface porosity is clear visible here via SEM thus verifying the reasons for samples to undergo Activation process using potassium hydroxide and Treatment using nitric acid, HNO3.

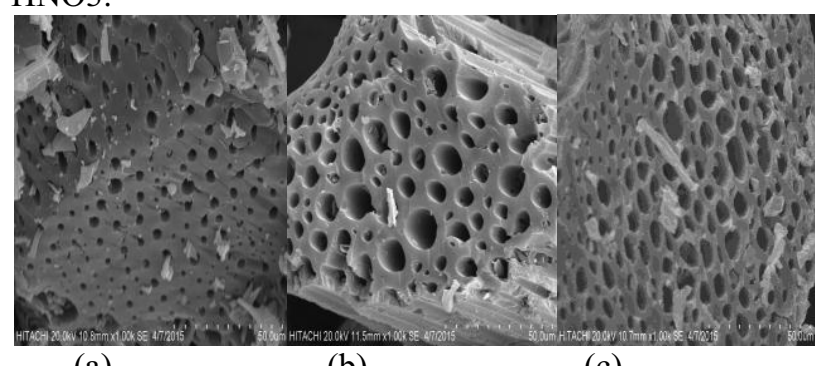

(a) (b)

(c)

Figure 2. SEM images of (a) Original PKS Biochar, (b) activated PKS biochar and (c) treated PKS biochar

Table 1 shows the overall Raman spectrum comparison of PKS Biochar samples. The G-band peaks recorded for Original, Activated, and Treated samples are $1583.14 \mathrm{~cm}-1, \quad 1578.82 \mathrm{~cm}^{-1}$, and $1582.81 \mathrm{~cm}^{-1}$ respectively. The peaks that appear for the D-band are
$1354.39 \mathrm{~cm}-1,1354.06 \mathrm{~cm}-1$ and $1354.72 \mathrm{~cm}-1$ for the same samples of Biochar.

Table 1. Overall Comparison of Biochar Samples

\begin{tabular}{|l|l|l|l|}
\hline Sample & $\begin{array}{l}\text { Original } \\
\text { Palm } \\
\text { Kernell } \\
\text { Shell } \\
\text { Biochar }\end{array}$ & $\begin{array}{l}\text { Activated } \\
\text { Palm } \\
\text { Kernell } \\
\text { Shell } \\
\text { Biochar }\end{array}$ & $\begin{array}{l}\text { Treated } \\
\text { Palm Kernell } \\
\text { Shell } \\
\text { Biochar }\end{array}$ \\
\hline D-Band (cm-1) & 1354.39 & 1354.06 & 1354.72 \\
\hline G-Band (cm-1) & 1583.14 & 1578.82 & 1582.81 \\
\hline (ID/IG) ratio & 0.856 & 0.858 & 0.857 \\
\hline
\end{tabular}

The ratio between the D-band and G-band intensities (ID/IG) represents the scale of defect concentration. The higher the intensity ratio, the higher the defect concentration. The defect mentioned here corresponds to the proportion of $\mathrm{sp}^{3}$ carbon formation, which caused disruptions to the original $\mathrm{sp}^{2}$ bonding, hence attributing to the structural defects being measure. In this case the values of $(\mathrm{ID} / \mathrm{IG})$ for Original, Activated and Treated samples are $0.856,0.858$, and 0.857 respectively. The increase in (ID/IG) after the introduction of nitric acid to treat the samples is due to the hydrothermal oxidation caused by nitric acid. This process generates oxygen containing functional groups on the surface of the sample thus increasing the number of $\mathrm{sp}^{3}$ hybridized carbon, causing increased surface defects.

Figure 3 illustrates the spectrum for raw, treated and activated PKS biochar sample. The peaks recorded for $\mathrm{C}=\mathrm{O}$ are $1590.71 \mathrm{~cm}-1,1591.39 \mathrm{~cm}-1$, and $1591.16 \mathrm{~cm}-1$ respectively which corresponds to the ketones, carboxylic acid, esters and anhydrides, respectively. The stretching that occurred at $1094.38 \mathrm{~cm}-1,1018.83 \mathrm{~cm}-1$ and $1243.54 \mathrm{~cm}-1$ can all be assigned to the $\mathrm{C}-\mathrm{N}$ stretching. The bands around 3700 which can be ascribed to O-H of phenol were not found on the Treated sample but appear on the raw and activated biochar at 3717.22 and 3747.85. This shows that there was a slight shift in Raman bands which was caused by the activation process using potassium hydroxide. Another clear shift in Raman bands occurred in the band range of $1018-1243 \mathrm{~cm}-1$. These bands are attributed to the $\mathrm{C}-\mathrm{N}$ stretching which varied from $1094.38 \mathrm{~cm}-1$ and $1018.83 \mathrm{~cm}-1$ for raw and activated biochar to $1243.54 \mathrm{~cm}-1$ for the treated biochar.

Another obvious change in bands was at band $1362.58 \mathrm{~cm}-1$ which was not present in the Raw biochar, appeared in Activated and Treated biochar although it appears to be shifted slight in the Treated biochar bands at $1243.54 \mathrm{~cm}-1$. This range of bands can be assigned to the $\mathrm{C}-\mathrm{O}$ stretching and $\mathrm{OH}$ bending of alcohol and carboxylic acids. 


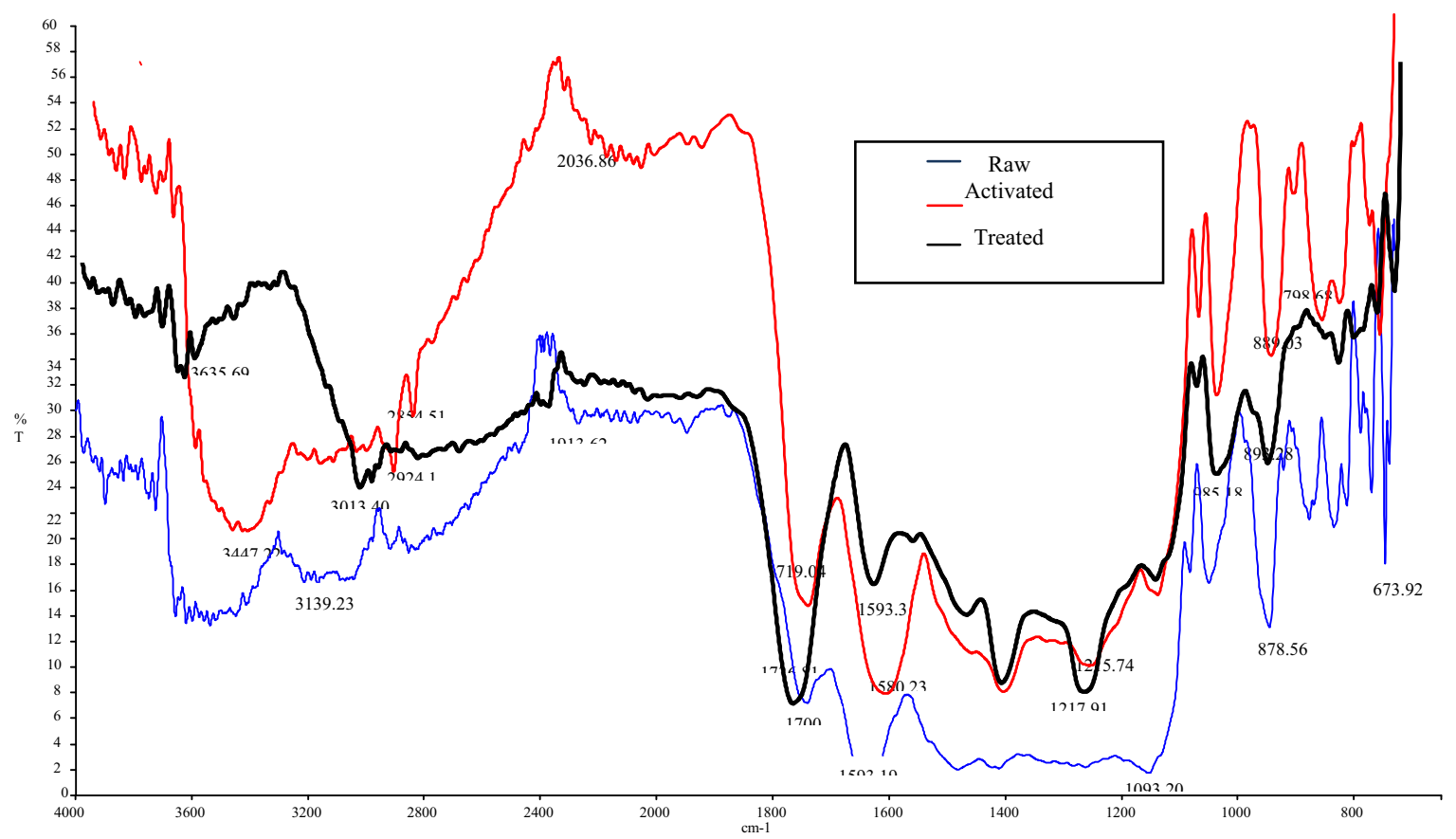

Figure 3. FTIR spectrum raw, activated and treated PKS biochars

In all, similar functional groups were observed on the surface of the activated carbons due to their exposure to similar chemical treatment and narrow variation of temperature of activation and treatment (see Table 2)

Table 2. Peak Assignments for Raw, Activated and Treated Biochar Samples.

\begin{tabular}{|l|l|l|l|}
\hline & Original & Activated & Treated \\
\hline $\begin{array}{l}\text { Aromatic C-H } \\
(\mathrm{cm}-1)\end{array}$ & 3015.23 & 3012.36 & 3010.93 \\
\hline $\begin{array}{l}\text { Aliphatic C-H } \\
(\mathrm{cm}-1)\end{array}$ & 2813.40 & 2812.74 & 2815.28 \\
\hline $\begin{array}{l}\mathrm{C}=\mathrm{O} \text { stretching } \\
(\mathrm{cm}-1)\end{array}$ & 1704.86 & 1713.65 & 1721.86 \\
\hline $\begin{array}{l}\mathrm{C}=\mathrm{C} \text { stretching } \\
(\mathrm{cm}-1)\end{array}$ & 1590.71 & 1591.39 & 1591.16 \\
\hline $\begin{array}{l}\text { O-H stretching } \\
(\mathrm{cm}-1)\end{array}$ & 3717.22 & 3747.85 & 3648.66 \\
\hline $\begin{array}{l}\text { C-N stretching } \\
(\mathrm{cm}-1)\end{array}$ & 1094.38 & 1018.83 & 1243.54 \\
\hline
\end{tabular}

The values of BET specific surface area are 0.2982 12.9034 and 53.8346 and for original, activated and treated biochar, respectively. The average pore size of the samples also decreased from 338.19797 to $51.78124 \mathrm{~nm}$ after potassium hydroxide, $\mathrm{KOH}$ activation. The pore size for Treated biochar increased drastically to $7929.64 \mathrm{~nm}$. This has been verified by the SEM results obtained that clearly shows the larger pore sizes on the biochar after treatment with nitric acid.

\subsection{Cyclic Voltammetry (CV)}

The suitability, of the carbon obtained for electrodes in a supercapacitor was evaluated by using an electrochemical technique, cyclic voltammetry $(\mathrm{CV})$. With the prepared Activated biochar as the electrodes, the electrochemical behavior of the biochar was characterized with the $\mathrm{CV}$ using $0.5 \mathrm{~mol}$ potassium hydroxide as the electrolyte and $\mathrm{SCE}$ as reference electrode. The $\mathrm{CV}$ response of the electrodes was measured at different scan rates varying from 10 to $200 \mathrm{mV} \mathrm{s} 1$ at potentials between 1 and $0 \mathrm{~V}$. Figure 4 shows CV curve of the biochar samples in 0.5 mol potassium hydroxide.

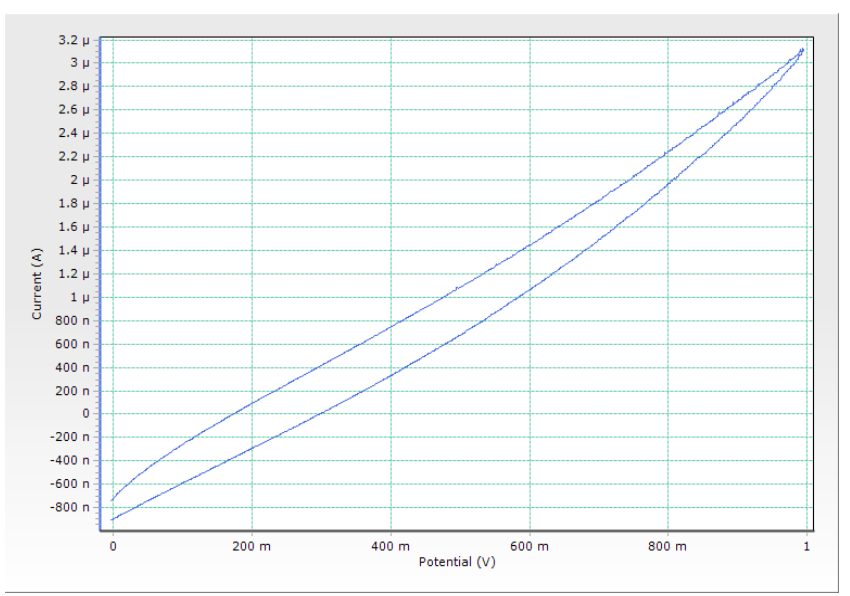

(a) 


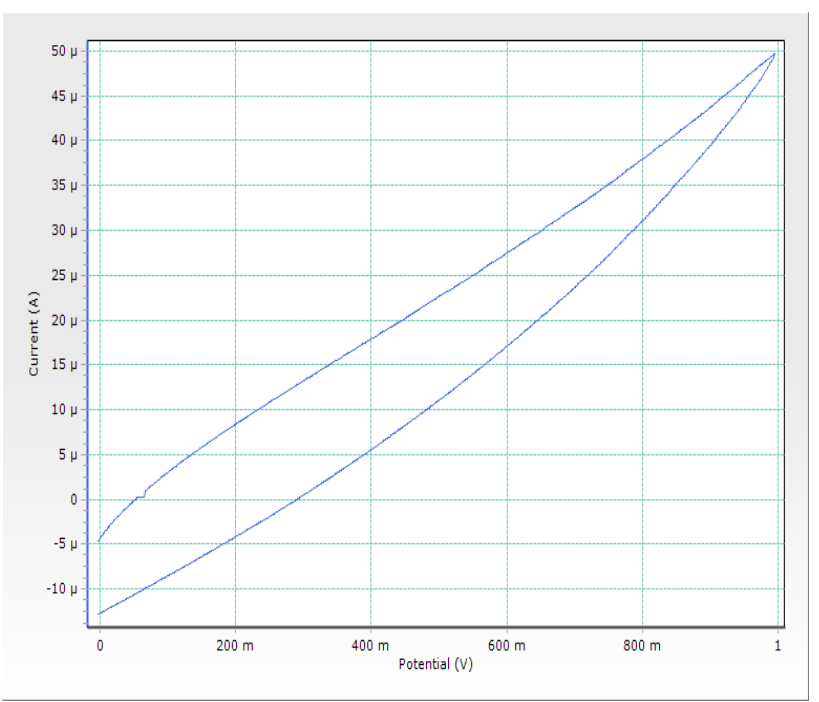

(b)

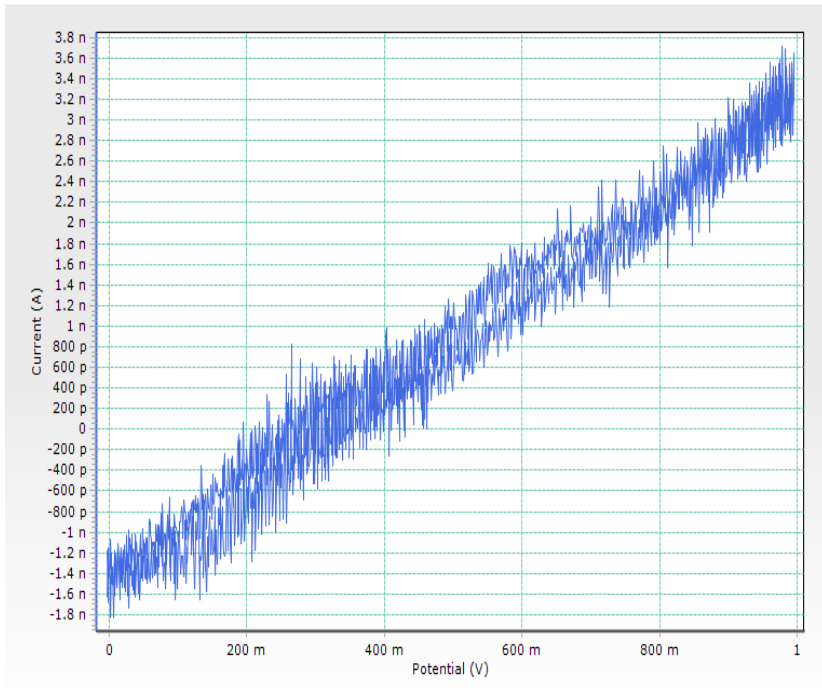

(c)

Figure 4. Cylic Voltammometry (CV) (a) raw, (b) activated and (c) treated PKS biochar electrodes at scanning rate $0.05 \mathrm{mV} / \mathrm{s}$

From these figures, it is observed that the Activated biochar has a better closer resemblance to a square shape compared to the original biochar $\mathrm{CV}$ curve. According to this observation, the activated biochar shows better capacitor characteristics as compared to the original biochar. The treated biochar however exhibits a very scattered CV curve due to the high level of noise. This can be attributed to the uneven rough surface of the treated electrode when tested using CV.

\section{Conclusion}

Sustainable production of activated carbon was produced from Palm Kernel Shell using potassium hydroxide as the activation agent and nitric acid as the treatment medium, was developed and the results reported. A supercapacitor has been developed using $0.5 \mathrm{~mol}$ Potassium hydroxide as the electrolyte. Based on the test results albeit not perfect the palm kernel shell based biochar has the potential to be a lower cost substitute for the advanced, more expensive carbon materials currently being commercialized such as metal carbides, graphene or activated graphene. The process of synthesizing the palm kernel shell biochar is without artificial templates hazardous substances.

\section{Acknowledgement}

Authors would like to acknowledge the financial support from Ministry of Education (MOE), Malaysia via FRGS grant (Program code:FRGS/1/2014/TK05/UPM/02/8)."

\section{References}

1. Xia, K., Gao, Q., Jiang, J. \& Hu, J., 2008. Hierarchical porous carbons with controlled micropores and mesopores for supercapacitor electrode materials. Carbon, Volume 46, pp. 17181726.A. Mecke, I. Lee, J.R. Baker jr., M.M. Banaszak Holl, B.G. Orr, Eur. Phys. J. E 14, 7 (2004)

2. Halper, S. M. \& Ellenbogen, C. J., 2006. Supercapacitor: A Brief Overview, Virginia: The MITRE Corporation.

3. Pandolfo, A. G. \& Hollenkamp, A. F., 2006. Carbon properties and their role in supercapacitors. Journal of Power Sources, 157(1), pp. 11-27.

4. Jin, H., Wang, X., Gu, Z. \& Polin, J., 2013. Carbon materials from high ash biochar for supercapacitor and improvement. Journal of Power Sources, Volume 236, pp. 285-292.

5. Junhua Jianga,*, Lei Zhangb, Xinying Wanga, Nancy Holma, Kishore Rajagopalana,Fanglin Chenb, Shuguo Ma, 2013. Highly ordered macroporous woody biochar with ultra-high carbon content as supercapacitor electrodes. Electrochimica Acta, Volume 113, pp. 481-489.

6. Kötz, R. \& Carlen, M., 2000. Principles and applications of electrochemical capacitors. Electrochimica Acta, p. 2483-2498.

7. Zhu, Y., 2011. Carbon-Based Supercapacitors Produced by Activation of Graphene. Science, Volume 332, pp. 1537-1539.

8. Balathanigaimani, M.., 2008. Highly porous electrodes from novel corn grains-based activated carbons for electrical double layer capacitors. Electrochemistry Communications, 10(6), pp. 868871.

9. Ito, E., 2007. Nanoporous carbons from cypress II . Application to electric double layer capacitors. New Carbon Materials, 22(4), pp. 321-326

10. Anjos, D. M., 2013. Pseudocapacitance and performance stability of quinone-coated carbon onions. Nano Energy, 2(5), pp. 702-712

11. Nor, N. S. M., 2015. Influence of gamma irradiation exposure on the performance of supercapacitor electrodes made from oil palm empty fruit bunches. Energy, Volume 79, pp. 183-194.

12. Kuratani, K., 2011. Converting rice husk activated carbon into active material for capacitor using threedimensional porous current collector. Journal of Power Sources, 196(24), pp. 10788-10790. 
13. Sahu, V., 2015. Heavily nitrogen doped, graphene supercapacitor from silk cocoon. Electrochimica Acta, Volume 160, pp. 244-253

14. Li, X. et al., 2011. Bioresource Technology Preparation of capacitor's electrode from sunflower seed shell. Bioresource Technology, 102(2), pp. 1118-1123.
15. Wan azlina Wan Ab Karim Ghani, Gabriel da-Silva and azil bahari Alias (2014). Physico-chemical characterizations of sawdust-derived biochar as potential solid fuels. Malaysian journal of analytical science, 18 (3), 724-729. 